\title{
3 Research Square \\ Relieff Matching Feature Selection for Emotion Recognition Based on EEG Signal
}

\section{Xiaodan Zhang ( $\nabla$ zhangxiaodan@xpu.edu.cn )}

School of Electronic Information, Xi'an Polytechnic University Xi 'an 710600, China; https://orcid.org/0000-0001-7581-7945

\section{Tao Li}

Xi'an Polytechnic University

\section{Yichong She}

Xidian University

\section{Rui Zhao}

Xi'an Polytechnic University

Jinxiang Du

Xi'an Polytechnic University

\section{Yawen Zhai}

Xi'an Polytechnic University

\section{Xizheng Ke}

Xi'an University of Technology

\section{Research}

Keywords: emotion recognition, RMFS, wavelet packet decomposition, empirical mode decomposition, support vector match

Posted Date: September 30th, 2020

DOI: https://doi.org/10.21203/rs.3.rs-80078/v1

License: (c) (i) This work is licensed under a Creative Commons Attribution 4.0 International License.

Read Full License 


\section{Abstract}

ReliefF Matching Feature Selection (RMFS) is proposed in the paper, which can solve the problem of individual specificity and global threshold mismatch of emotion recognition. Firstly, EEG was decomposed into six emotion-related bands by wavelet packet, then EMD was employed for extracting the 10 categories of features of wavelet coefficient and IMF component of the reconstructed signal; Secondly, the optimization formula of the feature group weight was proposed based on feature sets selected by ReliefF, and it can get the weights of different test features, which were the global optimal matching feature group and the corresponding matching channel, so it can eliminate the redundant information and solve the problem of individual specificity. Finally, SVM was employed to identify the test feature group data to obtain emotional recognition results. The experimental results show that the average correct rates of RMFS for two-category of the valence and the arousal are $93.28 \%$ and $93.32 \%$, and the four-categories are higher than $83 \%$. The efficiency of the single subject using RMFS is improved by $42.65 \%$, which is better than the traditional ReliefF algorithm.

\section{Full Text}

This preprint is available for download as a PDF.

\section{Figures}




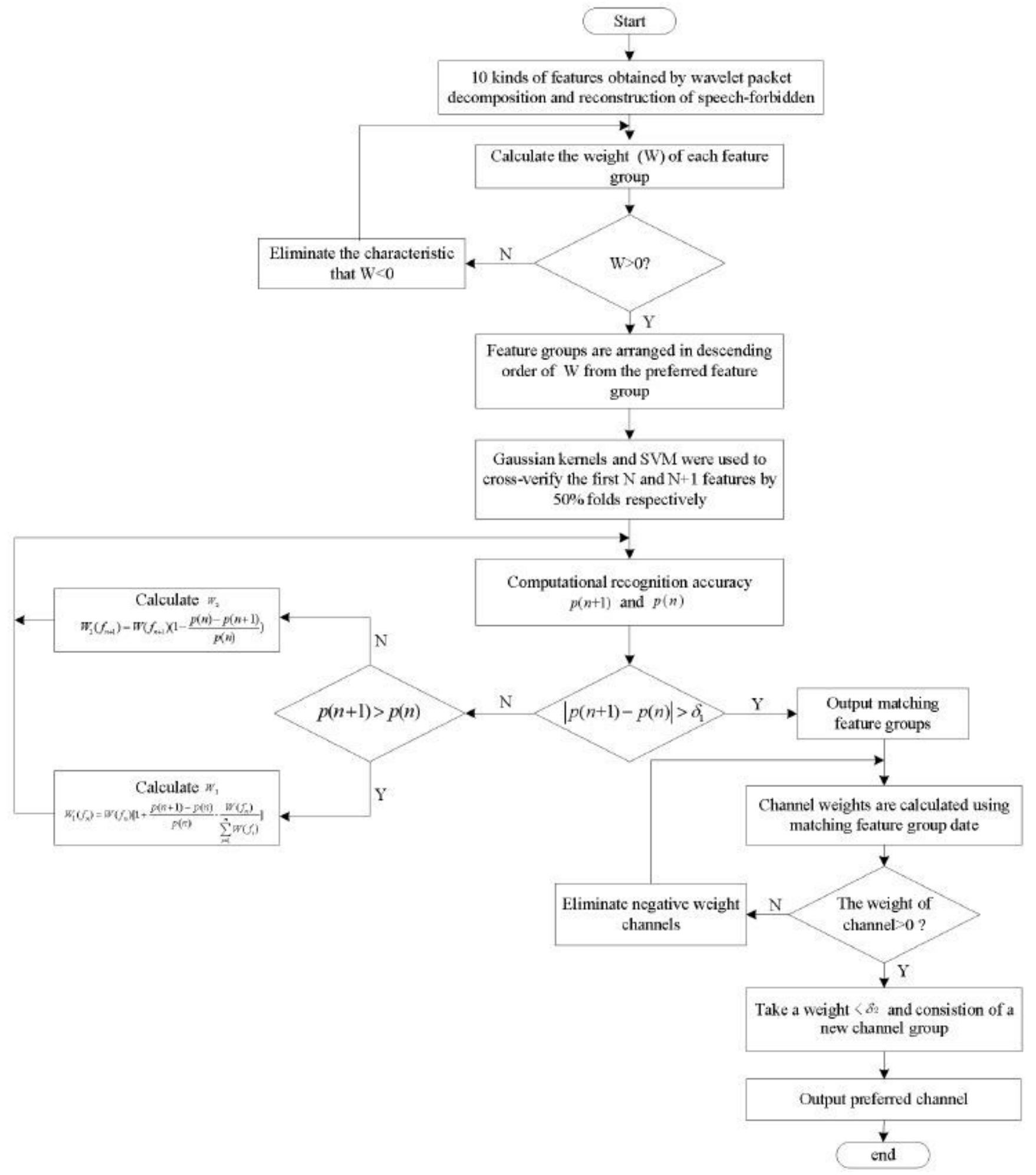

Figure 1

The flow chart of RMFS 


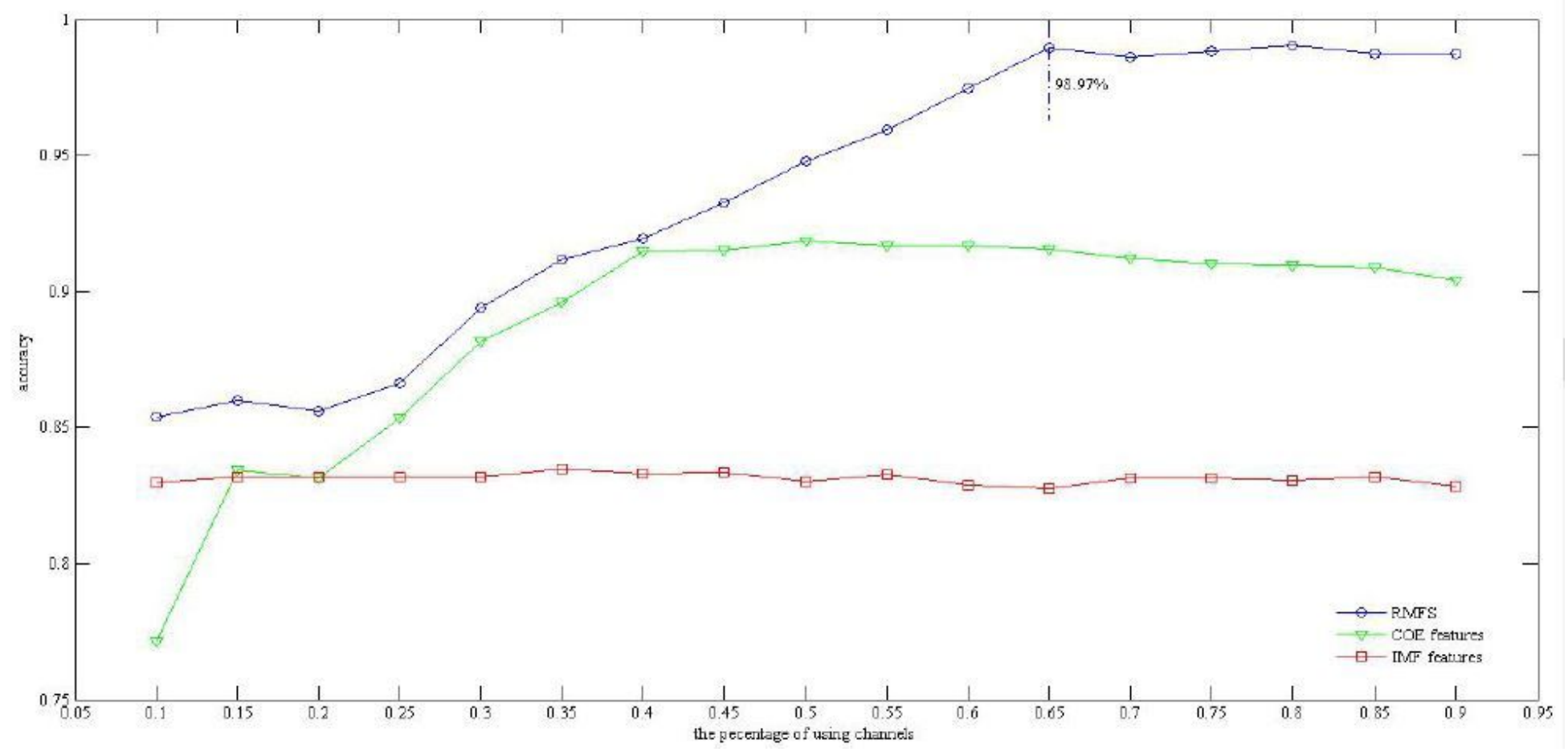

Figure 2

s25 potency two classification channel selection result

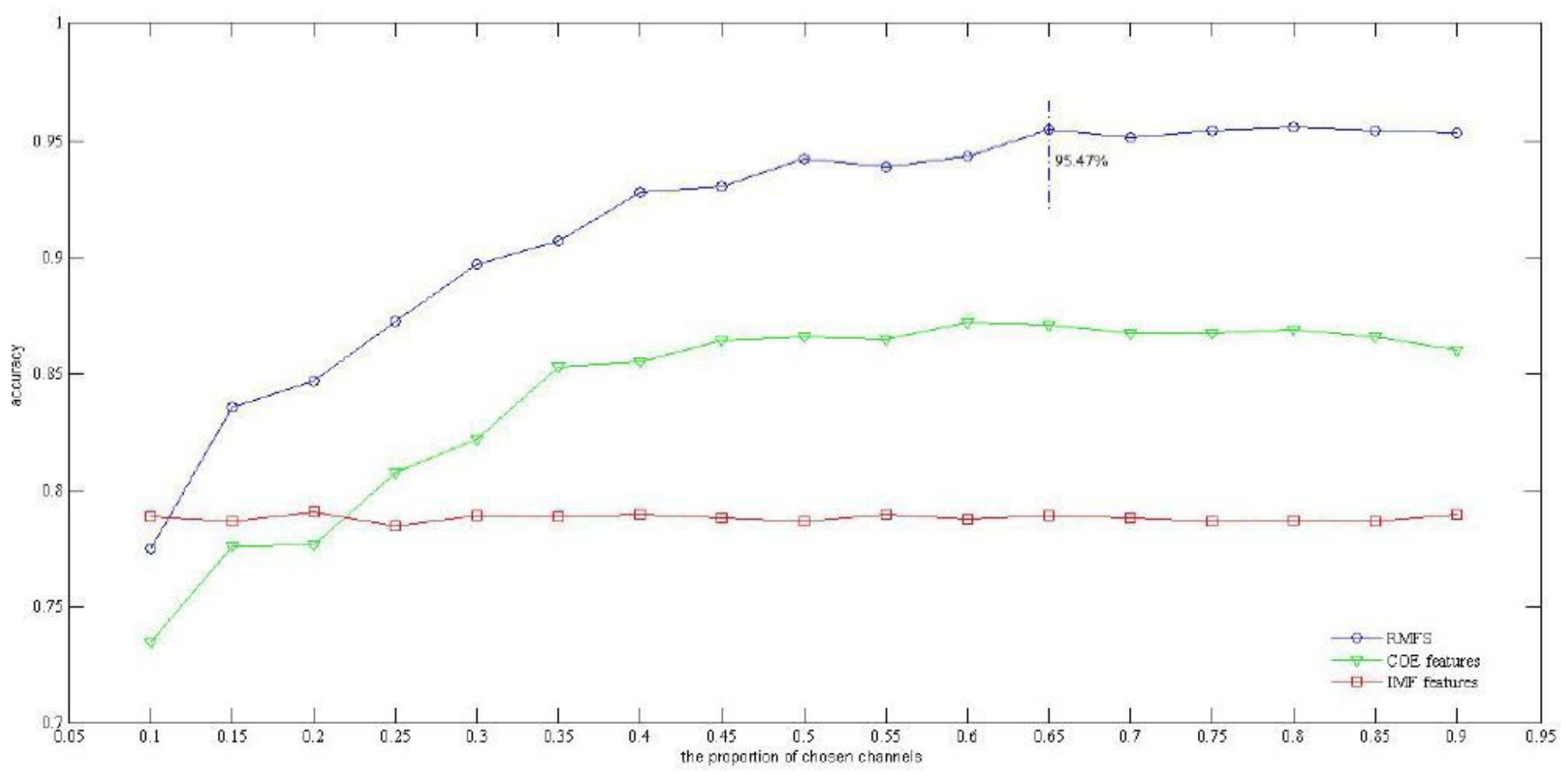

Figure 3

s25 wake-up two classification channel selection results 


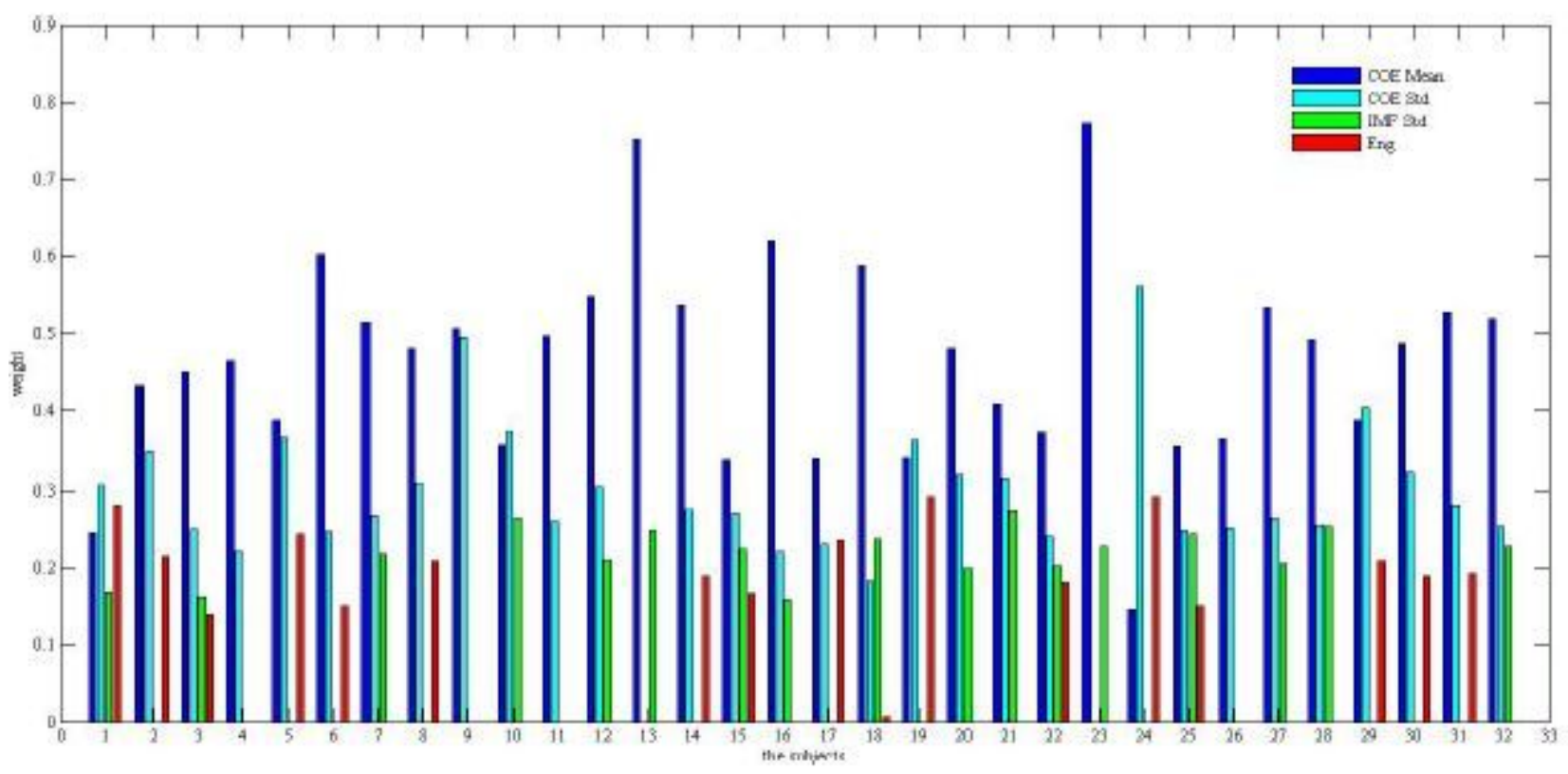

Figure 4

Composition of 32-bit subject titer two-category matching feature group and its weight

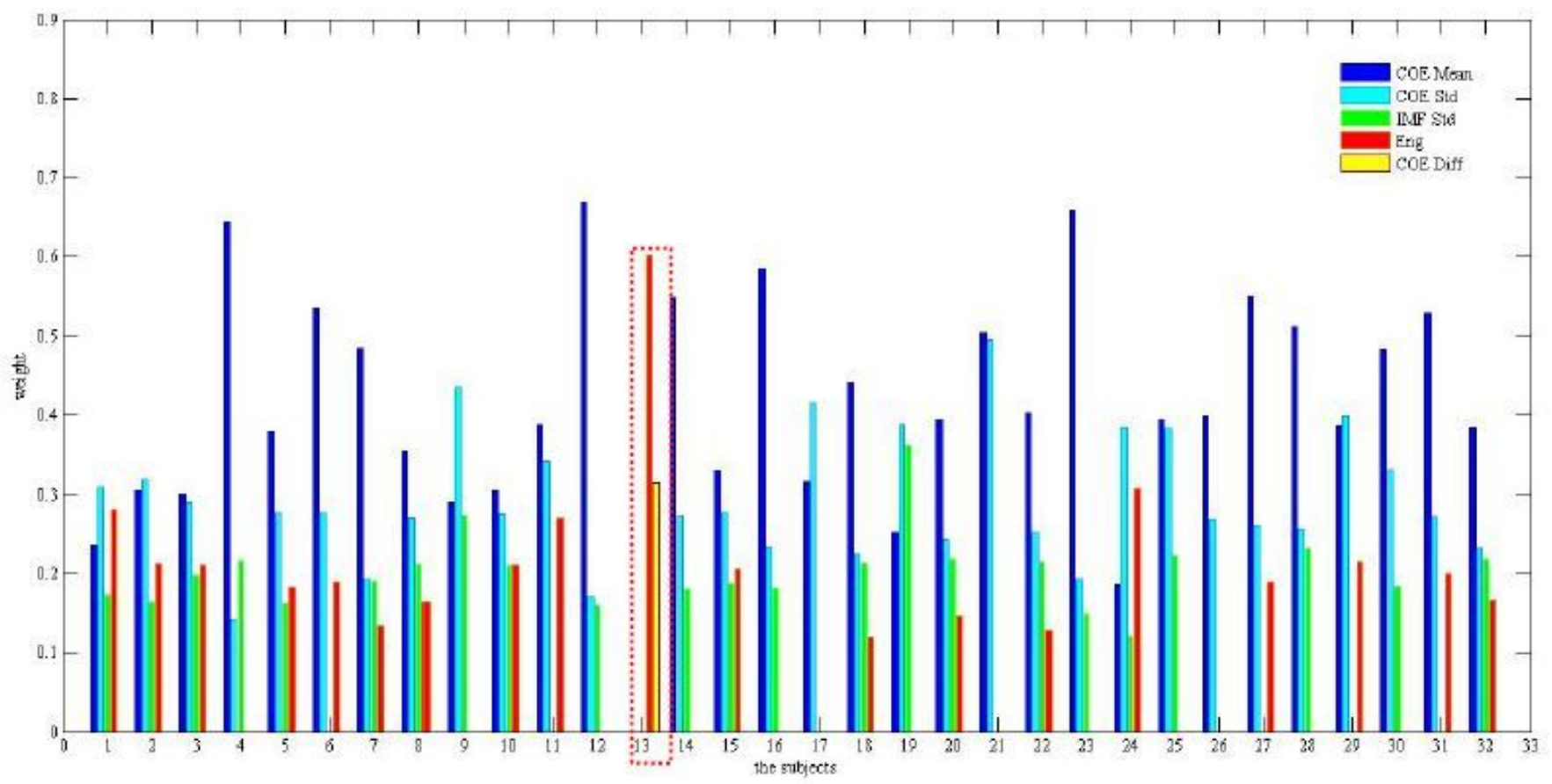

Figure 5

Composition of 32-bit subjects' arousal degree binary classification matching feature group and its weight 


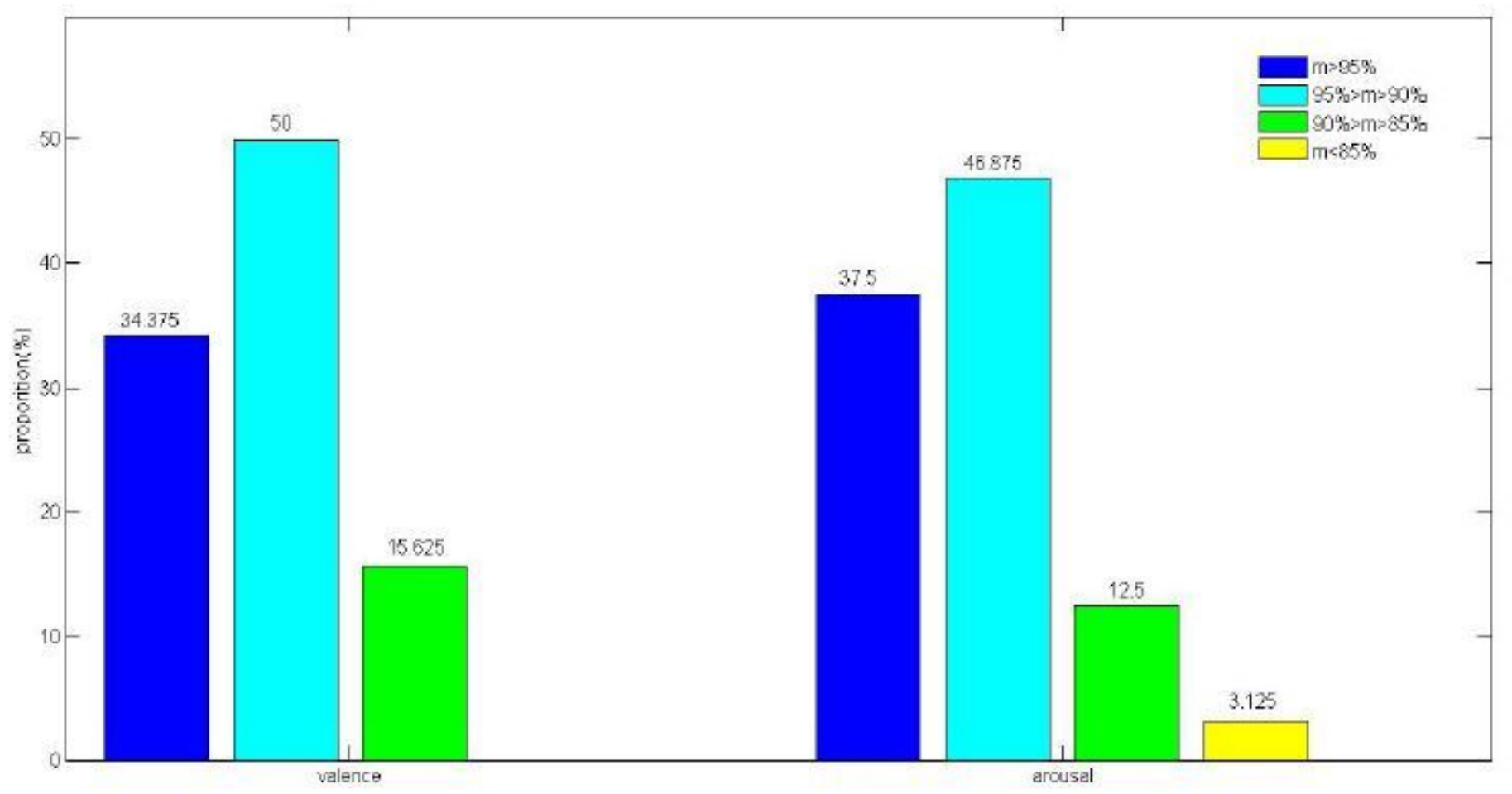

Figure 6

Statistical results of sentiment classification

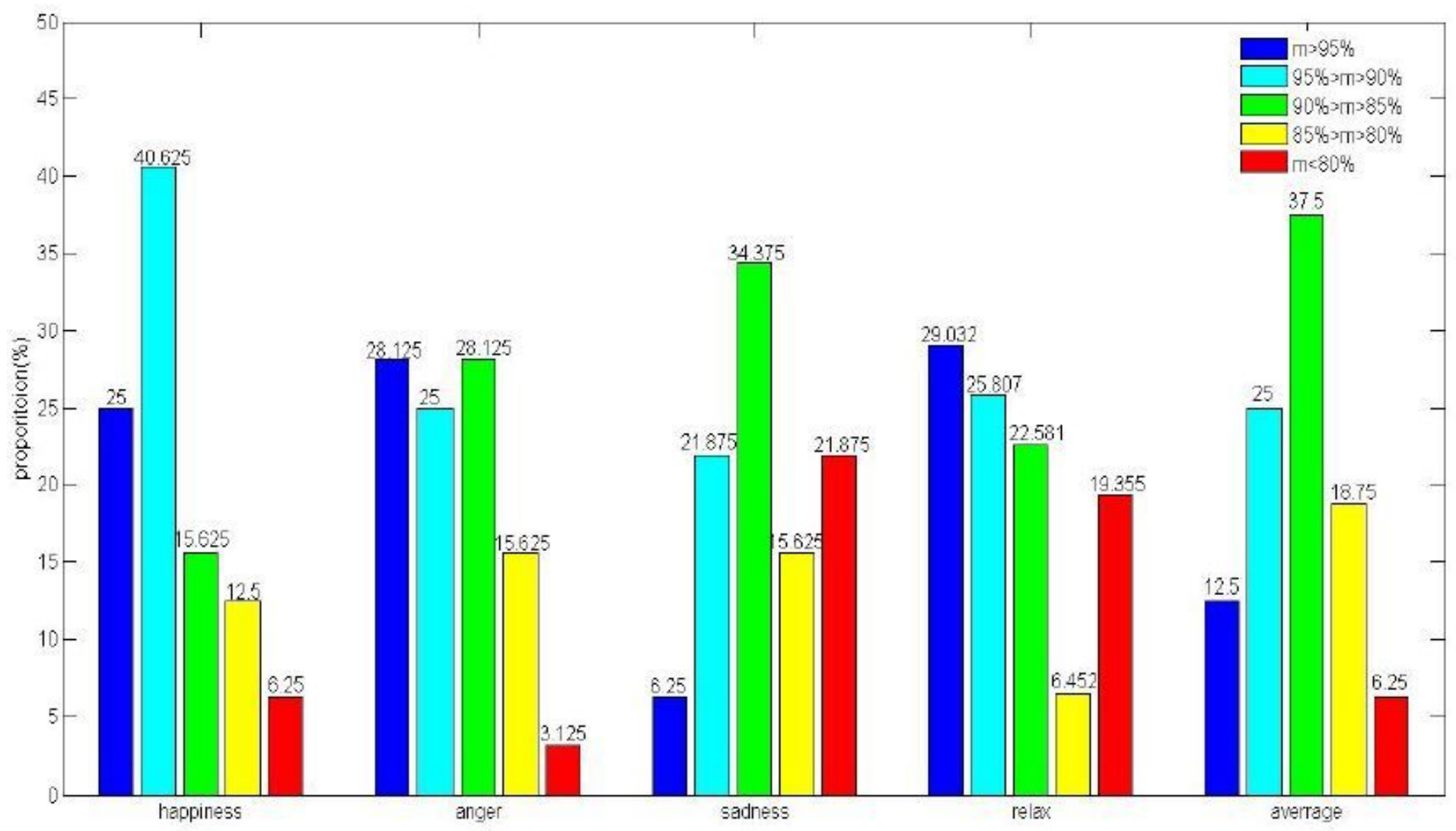

Figure 7 
Statistical results of emotion classification 